International Journal of Pure and Applied Mathematics

Volume 103 No. 2 2015, 321-332

ISSN: 1311-8080 (printed version); ISSN: 1314-3395 (on-line version)

url: http://www.ijpam.eu

doi: http://dx.doi.org/10.12732/ijpam.v103i2.16

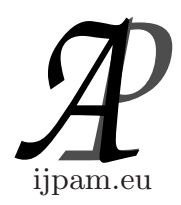

\title{
A NOTE ON SPACELIKE CURVES ACCORDING TO TYPE-2 BISHOP FRAME IN MINKOWSKI 3-SPACE $E_{1}^{3}$
}

\author{
Süha Yılmaz ${ }^{1}$, Yasin Ünlütürk ${ }^{2} \S$ \\ ${ }^{1}$ Buca Faculty of Education \\ Dokuz Eylül University \\ 35150, Buca-Izmir, TURKEY \\ ${ }^{2}$ Department of Mathematics \\ Kırklareli University \\ 39000, Kırklareli, TURKEY
}

\begin{abstract}
In this work, we give some characterizations of spacelike curve with spacelike principal normal vector according to type-2 Bishop frame in Minkowski 3-space $E_{1}^{3}$. Also, we obtain position vector of spacelike curves on Lorentzian sphere with respect to the type-2 Bishop curvatures. Finally, we establish some relations among Frenet apparatus of an evolute curve and type2 Bishop apparatus of an involute curve in Minkowski 3-space.
\end{abstract}

AMS Subject Classification: 53A35, 53A40, 53B25

Key Words: Minkowski 3-space, involute-evolute curves couple, inclined curve, type-2 Bishop apparatus

\section{Introduction}

Bishop frame has applications in the areas of biology and computer graphics. It may be possible to compute information about the shape of sequences of DNA using a curve defined by the Bishop frame. The Bishop frame may also provide a new way to control virtual cameras in computer animations. It is desirable to

Received: June 3, 2015

(c) 2015 Academic Publications, Ltd.

$\S_{\text {Correspondence author }}$ url: www.acadpubl.eu 
have the camera gaze direction pointing forward along a space curve throughout the motion (see [3]).

The construction of the Bishop frame is due to L. R. Bishop in (see [1]). That is why he defined this frame that curvature may vanish at some points on the curve. That is, second derivative of the curve may be zero. In this situation, an alternative frame is needed for non continously differentiable curves on which Bishop (parallel transport frame) frame is well defined and constructed in Euclidean and its ambient spaces (see [7], [11], [13], [15]). The advantages of Bishop frame, and comparisons of Bishop frame with the Frenet frame in Euclidean 3-space were given by Bishop (see [1]).

In theory of curves, the related curves for which there exist corresponding relations between the curves are very interesting and important topics. One of the most fascinating examples of such curves are involute-evolute curves whose tangents are orthogonal to each other. C. Huygens, who is also known for his works in optics, discovered involutes while trying to build a more accurate clock. And, in particular, involute-evolute curve couple, inclined curves and W-curves are well known concepts in the classical differential geometry (see [9], [11]). Also the relations between Frenet frames of spacelike involute-evolute couple of curves were given in Minkowski space-time (see [10]). Additionally, inclined curves were characterised in Minkowski space-time by the same authors (see $[12])$.

In this work, we give some characterizations of spacelike curve with spacelike principal normal vector according to type-2 Bishop frame in Minkowski 3-space $E_{1}^{3}$. Also, we obtain position vector of spacelike curves on Lorentzian sphere with respect to the type-2 Bishop curvatures. Finally, we establish some relations among Frenet apparatus of an evolute curve and type- 2 Bishop apparatus of an involute curve in Minkowski 3-space.

\section{Preliminaries}

The fundamentals of Minkowski 3-space below is cited from (see [4], [6]).

The three dimensional Minkowski space $E_{1}^{3}$ is a real vector space $R^{3}$ endowed with the standard flat Lorentzian metric given by

$$
\langle,\rangle_{L}=-d x_{1}^{2}+d x_{2}^{2}+d x_{3}^{2}
$$

where $\left(x_{1}, x_{2}, x_{3}\right)$ is a rectangular coordinate system of $E_{1}^{3}$. This metric is an indefinite one. 
Let $u=\left(u_{1}, u_{2}, u_{3}\right)$ and $v=\left(v_{1}, v_{2}, v_{3}\right)$ be arbitrary vectors in $E_{1}^{3}$, the Lorentzian cross product of $u$ and $v$ is defined as

$$
u \times v=-\operatorname{det}\left[\begin{array}{ccc}
-i & j & k \\
u_{1} & u_{2} & u_{3} \\
v_{1} & v_{2} & v_{3}
\end{array}\right]
$$

Recall that a vector $v \in E_{1}^{3}$ can have one of three Lorentzian causal characters: it is a spacelike vector if $\langle v, v\rangle>0$ or $v=0$; timelike $\langle v, v\rangle<0$ and null (lightlike) $\langle v, v\rangle=0$ for $v \neq 0$. Similarly, an arbitrary curve $\delta=\delta(s)$ in $E_{1}^{3}$ can locally be spacelike, timelike or null (lightlike) if its velocity vector $\delta^{\prime}$ are ,respectively, spacelike, timelike or null (lightlike), for every $s \in I \subset \mathbb{R}$. The pseudo-norm of an arbitrary vector $a \in E_{1}^{3}$ is given by $\|a\|=\sqrt{|\langle a, a\rangle|}$. The curve $\delta=\delta(s)$ is called a unit speed curve if its velocity vector $\delta$ is unit one i.e., $\left\|\delta^{\prime}\right\|=1$. For vectors $v, w \in E_{1}^{3}$, they are said to be orthogonal eachother if and only if $\langle v, w\rangle=0$. Denote by $\{T, N, B\}$ the moving Serret-Frenet frame along the curve $\delta=\delta(s)$ in the space $E_{1}^{3}$.

For an arbitrary spacelike curve $\delta=\delta(s)$ in $E_{1}^{3}$, the Serret-Frenet formulae are given as follows

$$
\left[\begin{array}{l}
T^{\prime} \\
N^{\prime} \\
B^{\prime}
\end{array}\right]=\left[\begin{array}{ccc}
0 & \kappa & 0 \\
\gamma \kappa & 0 & \tau \\
0 & \tau & 0
\end{array}\right]\left[\begin{array}{l}
T \\
N \\
B
\end{array}\right]
$$

where $\gamma=\mp 1$, and the functions $\kappa$ and $\tau$ are respectively the first and second (torsion) curvature. $T(s)=\delta^{\prime}(s), N(s)=\frac{T^{\prime}(s)}{\kappa(s)}, B(s)=T(s) \times N(s)$ and $\tau(s)=\frac{\operatorname{det}\left(\delta^{\prime}, \delta^{\prime \prime}, \delta^{\prime \prime \prime}\right)}{\kappa^{2}(s)}$.

If $\gamma=-1$, then $\delta(s)$ is a spacelike curve with spacelike principal normal $N$ and timelike binormal $B$, its Serret-Frenet invariants are given as

$$
\kappa(s)=\sqrt{\left\langle T^{\prime}(s), T^{\prime}(s)\right\rangle} \text { and } \tau(s)=-\left\langle N^{\prime}(s), B(s)\right\rangle .
$$

If $\gamma=1$, then $\delta(s)$ is a spacelike curve with timelike principal normal $N$ and spacelike binormal $B$, also we obtain its Serret-Frenet invariants as

$$
\kappa(s)=\sqrt{-\left\langle T^{\prime}(s), T^{\prime}(s)\right\rangle} \text { and } \tau(s)=\left\langle N^{\prime}(s), B(s)\right\rangle .
$$

The Lorentzian sphere $S_{1}^{2}$ of radius $r>0$ and with the center in the origin of the space $E_{1}^{3}$ is defined by

$$
S_{1}^{2}(r)=\left\{p=\left(p_{1}, p_{2}, p_{3}\right) \in E_{1}^{3}:\langle p, p\rangle=r^{2}\right\} .
$$


Theorem 1. ,(see [15]), Let $\delta=\delta(s)$ be spacelike unit speed curve with a spacelike principal normal. If $\left\{\Omega_{1}, \Omega_{2}, B\right\}$ is adapted frame, then we have

$$
\left[\begin{array}{l}
\Omega_{1}^{\prime} \\
\Omega_{2}^{\prime} \\
B^{\prime}
\end{array}\right]=\left[\begin{array}{ccc}
0 & 0 & \xi_{1} \\
0 & 0 & -\xi_{2} \\
-\xi_{1} & -\xi_{2} & 0
\end{array}\right]\left[\begin{array}{c}
\Omega_{1} \\
\Omega_{2} \\
B
\end{array}\right]
$$

Theorem 2. Let $\{T, N, B\}$ and $\left\{\Omega_{1}, \Omega_{2}, B\right\}$ be Frenet and Bishop frames, respectively. There exists a relation between them as

$$
\left[\begin{array}{l}
T \\
N \\
B
\end{array}\right]=\left[\begin{array}{ccc}
\sinh \theta(s) & \cosh \theta(s) & 0 \\
\cosh \theta(s) & \sinh \theta(s) & 0 \\
0 & 0 & 1
\end{array}\right]\left[\begin{array}{c}
\Omega_{1} \\
\Omega_{2} \\
B
\end{array}\right]
$$

where $\theta$ is the angle between the vectors $N$ and $\Omega_{1}$. Also, the type-2 Bishop curvatures are as follows:

$$
\xi_{1}=\tau(s) \cosh \theta(s), \xi_{2}=\tau(s) \sinh \theta(s) .
$$

The frame $\left\{\Omega_{1}, \Omega_{2}, B\right\}$ is properly oriented, and $\tau$ and

$$
\theta(s)=\int_{0}^{s} \kappa(s) d s
$$

are polar coordinates for the curve $\delta=\delta(s)$. We shall call the set

$$
\left\{\Omega_{1}, \Omega_{2}, B, \xi_{1}, \xi_{2}\right\}
$$

as type-2 Bishop invariants of the curve $\delta=\delta(s)$ in $E_{1}^{3}$ (see [15]).

Definition 3. Let $\delta$ be a spacelike curve in $E_{1}^{3}$ and $V_{1}$ be the first Frenet vector field of $\delta$. If

$$
\left\langle V_{1}, X\right\rangle=\cosh \theta(\text { constant })
$$

for a constant unit vector field $X \in \chi\left(E_{1}^{3}\right)$, then $\delta$ is called a general helix (inclined curve) in $E_{1}^{3}$ ( see [2]).

Practically, the condition for a curve to be an inclined curve is that its tangent vector field has to form a constant angle with a constant vector field.

Definition 4. Let $\varphi$ and $\delta$ be two regular curves defined on an interval $(a, b) . \delta$ is an involute of $\varphi$ if $\delta\left(s_{0}\right)$ lies on the tangent line to $\varphi$ at $\varphi\left(s_{0}\right)$ and the tangents to $\varphi$ and $\delta$ at $\varphi\left(s_{0}\right)$ and $\delta\left(s_{0}\right)$ are perpendicular each other. $\delta$ is an evolute of $\varphi$ if $\varphi$ is an involute of $\delta$ (see [5]). 
Definition 5. Let $c$ and $r>0$ be given and $f(s)=(\delta-c)^{2}$. We say that $\delta$ has $j$ th order spherical contact with the sphere of radius $r$ and center $c$ at $s=s_{0}$ if

$$
f\left(s_{0}\right)=r^{2}, f^{\prime}\left(s_{0}\right)=f^{\prime \prime}\left(s_{0}\right)=\ldots=f^{(j)}\left(s_{0}\right)=0 .
$$

Note that the larger $j$ is, the closer $\mathrm{f}(s)$ is to being a constant function and so the closer $\delta$ is to lying on a sphere of radius $r$ and center $m$ (see [14]).

\section{Main Results}

In this part of the work, a regular spacelike curve with non-vanishing FrenetSerret curvatures is taken into consideration according to type-2 Bishop frame in $E_{1}^{3}$. In the classical theory of curves in Euclidean and its ambient spaces, there is a well-known concept that defines a curve of constant slope or general helix by the property that the tangent lines make a constant angle with a fixed direction.

The necessary and sufficient condition for a curve to be general helix in $E_{1}^{3}$ is that ratio of curvature to torsion has to be constant, (see [4]) that is,

$$
\frac{\kappa}{\tau}=\text { const }
$$

First we show this criteria in terms of the type-2 Bishop curvatures. Thus using (4) and (5), we give the following results:

Theorem 6. Let $\delta=\delta(s)$ be a regular spacelike curve with the curvatures $\kappa \neq 0$ and $\tau \neq 0$.(i) The curve $\delta$ is a general helix in $E_{1}^{3}$ if and only if its type- 2 Bishop curvatures satisfy

$$
\frac{\left(\frac{\xi_{2}}{\xi_{1}}\right)^{\prime} \xi_{1}^{2}}{\left(\xi_{1}^{2}-\xi_{2}^{2}\right)^{\frac{3}{2}}}=\text { const } \text {. }
$$

(ii) The curve $\delta$ is a helix in $E_{1}^{3}$ if and only if its type-2 Bishop curvatures satisfy

$$
\frac{\left(\frac{\xi_{2}}{\xi_{1}}\right)^{\prime}}{1-\left(\frac{\xi_{2}}{\xi_{1}}\right)^{2}}=\text { const., } \quad \xi_{1}^{2}-\xi_{2}^{2}=\text { const. }
$$


Proof. By (4), we have

$$
\tau=\xi_{1}^{2}-\xi_{2}^{2}
$$

using (4) and (5), we obtain

$$
\kappa=\frac{\left(\frac{\xi_{2}}{\xi_{1}}\right)^{\prime}}{1-\left(\frac{\xi_{2}}{\xi_{1}}\right)^{2}} .
$$

Finally, substituting (10) and (11) into (7), we get the result in (8). (ii) is a special class of the result (8).

Theorem 7. There does not exist any regular spacelike curve with curvatures $\kappa \neq 0$ and $\tau \neq 0$ in $E_{1}^{3}$ whose the first vector field of the type-2 Bishop frame $\Omega_{1}$ forms a constant angle with a fixed direction.

Proof. Assume that there is a regular spacelike curve $\delta=\delta(s)$ with curvatures $\kappa \neq 0$ and $\tau \neq 0$ in $E_{1}^{3}$ whose the first vector field of the type-2 Bishop frame forms a constant angle with a fixed direction. So we have a relation as

$$
\Omega_{1} \cdot U=\cos \theta
$$

where $\mathrm{U}$ is a fixed direction (nonzero vector) and $\theta$ is a constant angle. Differentiating (12) gives

$$
\xi_{1} B . U=0
$$

therefore we compose the axis $U$ according to type-2 Bishop frame as follows

$$
U=u_{1} \Omega_{1}+u_{2} \Omega_{2},
$$

where $u_{i}$ for $1 \leq i \leq 2$ are real valued functions of $s$. Differentiating (13), we obtain

$$
u_{1}^{\prime} \Omega_{1}+u_{2}^{\prime} \Omega_{2}+\left(u_{1} \xi_{1}-u_{2} \xi_{2}\right) B=0 .
$$

From (14), we have

$$
\left\{\begin{array}{l}
u_{1}^{\prime}=0 \\
u_{2}^{\prime}=0 \\
u_{1} \xi_{1}-u_{2} \xi_{2}=0
\end{array}\right.
$$

Thus we have

$$
\frac{\xi_{2}}{\xi_{1}}=\text { const }
$$

which is a contradiction. 
Theorem 8. There does not exist any regular spacelike curve with curvatures $\kappa \neq 0$ and $\tau \neq 0$ in $E_{1}^{3}$ whose the second vector field of the type-2 Bishop frame $\Omega_{2}$ forms a constant angle with a fixed direction.

Proof. As similar to the proof of Theorem 3.2, it can be shown for the second vector field of the type- 2 Bishop frame $\Omega_{2}$.

Corollary 9. There is not a spacelike inclined curve according to type-2 Bishop frame in $E_{1}^{3}$

Proof. This result can be seen from Definition 2.3 and the proof of Theorem 3.2 .

Theorem 10. Let a spacelike regular curve $\delta=\delta(s)$ lie on the Lorentzian sphere $S^{2}$ with the center $c$ and the radius $r$. The position vector of $\delta$ can be written as

$$
\begin{aligned}
\delta(s)-c & =\frac{\cosh \theta}{\theta^{\prime}(s)} \Omega_{1}+\frac{\sinh \theta}{\theta^{\prime}(s)} \Omega_{2} \\
& +\frac{1}{\theta^{\prime}\left(\xi_{1} \cosh \theta-\xi_{2} \sinh \theta\right)}\left\{\frac{\theta^{\prime \prime}}{\theta^{\prime}}\left(\sinh ^{2} \theta+\cosh ^{2} \theta\right)+2 \sinh \theta \cosh \theta\right\} B
\end{aligned}
$$

Proof. Let us suppose $\delta=\delta(s)$ lying on the Lorentzian sphere $S^{2}$ with the center $c$ and the radius $r$. Then we write

$$
\langle\delta(s)-c, \delta(s)-c\rangle=r^{2},
$$

for each $s \in I \subset E_{1}^{3}$. The equation (18) has to satisfy contact condition in Definition 2.5. Differentiating (18), we get

$$
\langle T, \delta(s)-c\rangle=0
$$

which means that $T \perp \delta(s)-c$. Then we can compose the vector $\delta(s)-c$ with respect to the basis $\left\{\Omega_{1}, \Omega_{2}, B\right\}$ as

$$
\delta(s)-c=m_{1} \Omega_{1}+m_{2} \Omega_{2}+m_{3} B
$$

where $m_{i}$ for $1 \leq i \leq 3$ are arbitrary functions of $s$. Using the tangent vector $T$ in (3) for $\theta=\int_{0}^{s} \kappa d s$ and (20) in (19) we have

$$
\left\langle\Omega_{1} \sinh \theta+\Omega_{2} \cosh \theta, m_{1} \Omega_{1}+m_{2} \Omega_{2}+m_{3} B\right\rangle=0 .
$$


From (21), we obtain

$$
\frac{m_{1}}{m_{2}}=-\operatorname{coth} \theta
$$

Differentiating (17) gives

$$
\langle\kappa N, \delta(s)-c\rangle+1=0
$$

Substituting the principal normal vector $N$ in (3), (5) and (20) into (23), we find

$$
\theta^{\prime}(s)\left[m_{1} \cosh \theta+m_{2} \sinh \theta\right]+1=0
$$

so that using (22) in (24), we obtain

$$
\left\{\begin{array}{l}
m_{1}=\frac{\cosh \theta}{\theta^{\prime}(s)}, \\
m_{2}=\frac{\sinh \theta}{\theta^{\prime}(s)} .
\end{array}\right.
$$

Again differentiating (23) we have

$$
\left\langle\kappa^{\prime} N, \delta(s)-c\right\rangle+\left\langle\kappa N^{\prime}, \delta(s)-c\right\rangle=0,
$$

and also the derivative of the principal normal vector in (3) is computed as

$$
N^{\prime}=\Omega_{1} \sinh \theta+\Omega_{2} \cosh \theta+B\left(\xi_{1} \cosh \theta-\xi_{2} \sinh \theta\right) .
$$

Substituting (3), (20) and (27) into (26), we find

$$
m_{3}=\frac{1}{\theta^{\prime}\left(\xi_{1} \cosh \theta-\xi_{2} \sinh \theta\right)}\left\{\frac{\theta^{\prime \prime}}{\theta^{\prime}}\left(\sinh ^{2} \theta+\cosh ^{2} \theta\right)+2 \sinh \theta \cosh \theta\right\} .
$$

Finally, replacing the values of the arbitrary functions $m_{i}$ for $1 \leq i \leq 3$ obtained as in (25) and (28) into (20), we have the position vector of $\delta(s)-c$ as in (17).

Theorem 11. Let $\varphi$ and $\delta$ be unit speed spacelike curves and $\varphi$ be evolute of $\delta$. The Frenet apparatus $\left\{T_{\varphi}, N_{\varphi}, B_{\varphi}, \kappa_{\varphi}, \tau_{\varphi}\right\}$ of $\varphi$ is expressed

$$
\begin{aligned}
& T_{\varphi}=-B, \\
& N_{\varphi}=\frac{1}{(s-c) \kappa_{\varphi}} \Omega_{1}+\frac{\xi_{2}}{(s-c) \kappa_{\varphi} \xi_{1}} \Omega_{2}, \\
& B_{\varphi}=\frac{1}{(s-c) \kappa_{\varphi}} \Omega_{2}-\frac{\xi_{2}}{(s-c) \kappa_{\varphi} \xi_{1}} \Omega_{1},
\end{aligned}
$$


and

$$
\begin{aligned}
& \kappa_{\varphi}=\sqrt{\left|\left(\frac{1}{s-c}\right)^{2}+\left(\frac{\xi_{2}}{(s-c) \xi_{1}}\right)^{2}\right|} \\
& \tau_{\varphi}=\frac{1}{\left|\left(\frac{\xi_{2}}{(s-c) \xi_{1}}\right)^{2}-\left(\frac{1}{s-c}\right)^{2}\right|}\left\{\xi_{1}^{2}+\tau(c-s) \xi_{1} \xi_{1}^{\prime}+(c-s) \xi_{1}^{\prime}\right. \\
& \left.-\xi_{1}\right\}(c-s) \xi_{1} \xi_{2}-\left\{\xi_{1} \xi_{2}+(c-s)\left(\xi_{1} \xi_{2}\right)^{\prime}\right. \\
& \left.+\left[(c-s) \xi_{1}^{\prime}-\xi_{1}\right] \xi_{2}\right\}(c-s) \xi_{1}^{2} .
\end{aligned}
$$

according to type-2 Bishop apparatus $\left\{\Omega_{1}, \Omega_{2}, B, \xi_{1}, \xi_{2}\right\}$ of $\delta$.

Proof. By Definition 2.4 we write

$$
\varphi=\delta+\lambda \Omega_{1}
$$

Differentiating (31) gives

$$
\frac{d \varphi}{d s_{\varphi}} \frac{d s_{\varphi}}{d s}=\frac{d \delta}{d s}+\frac{d \lambda}{d s} \Omega_{1}+\lambda \Omega_{1}^{\prime} .
$$

From Definition 2.4, we have

$$
1+\frac{d \lambda}{d s}=0
$$

and the solution of (33) is found as

$$
\lambda=c-s
$$

where $c$ is a constant. Thus the equation (31) turns into

$$
\varphi=\delta+(c-s) \Omega_{1}
$$

Differentiating (34) we have

$$
T_{\varphi} \frac{d s_{\varphi}}{d s}=(c-s) \xi_{1} B
$$

and also by the norm of (35) we obtain

$$
T_{\varphi}=-B, \quad \frac{d s_{\varphi}}{d s}=-(c-s) \xi_{1}
$$

Let us write the derivative of the tangent vector field of $\varphi$ as

$$
T_{\varphi}^{\prime}=\dot{T}_{\varphi} \frac{d s_{\varphi}}{d s}
$$


which ",", ". denote the tangent vector field $T_{\varphi}$ with respect to $s$ and $s_{\varphi}$, respectively.Using (36) in (37), we obtain

$$
\dot{T}_{\varphi}=\frac{1}{s-c} \Omega_{1}+\frac{\xi_{2}}{(s-c) \xi_{1}} \Omega_{2}
$$

and from $\kappa_{\varphi}=\left\|\dot{T}_{\varphi}\right\|$ we have

$$
\kappa_{\varphi}=\sqrt{\left|\left(\frac{1}{s-c}\right)^{2}+\left(\frac{\xi_{2}}{(s-c) \xi_{1}}\right)^{2}\right|} .
$$

The principal normal and binormal vectors of evolute are computed as follows:

$$
N_{\varphi}=\frac{1}{(s-c) \kappa_{\varphi}} \Omega_{1}+\frac{\xi_{2}}{(s-c) \kappa_{\varphi} \xi_{1}} \Omega_{2}
$$

and

$$
B_{\varphi}=\frac{1}{(s-c) \kappa_{\varphi}} \Omega_{2}-\frac{\xi_{2}}{(s-c) \kappa_{\varphi} \xi_{1}} \Omega_{1}
$$

in terms of invariants of type-2 Bishop frame in $E_{1}^{3}$. By using $\tau_{\varphi}=-\left\langle N_{\varphi}, B_{\varphi}^{\prime}\right\rangle$, the torsion of the evolute curve $\varphi$ in terms of type- 2 Bishop curvatures is computed as

$$
\begin{aligned}
\tau_{\varphi}=\frac{1}{\left|\left(\frac{\xi_{2}}{(s-c) \xi_{1}}\right)^{2}-\left(\frac{1}{s-c}\right)^{2}\right|}\left\{\xi_{1}^{2}+\tau(c-s) \xi_{1} \xi_{1}^{\prime}+(c-s) \xi_{1}^{\prime}-\xi_{1}\right\}(c-s) \xi_{1} \xi_{2} \\
-\left\{\xi_{1} \xi_{2}+(c-s)\left(\xi_{1} \xi_{2}\right)^{\prime}+\left[(c-s) \xi_{1}^{\prime}-\xi_{1}\right] \xi_{2}\right\}(c-s) \xi_{1}^{2}
\end{aligned}
$$

where

$$
B_{\varphi}^{\prime}=\left[-\frac{\xi_{2}}{(s-c) \kappa_{\varphi} \xi_{1}}\right]^{\prime} \Omega_{1}+\left[\frac{1}{(s-c) \kappa_{\varphi}}\right]^{\prime} \Omega_{2}+\left[-\frac{2 \xi_{2}}{(s-c) \kappa_{\varphi}}\right] B
$$

Corollary 12. Let $\varphi$ and $\delta$ be unit speed space-like curves and $\varphi$ be an evolute of $\delta$ according to type-2 Bishop frame in $E_{1}^{3}$. The evolute $\varphi$ is never an inclined curve in $E_{1}^{3}$.

Proof. This is a trivial result of Theorem 3.2. 


\section{References}

[1] L.R. Bishop, There is more than one way to frame a curve, Amer. Math. Month,. 82 (1975) 246-251, doi: 10.2307/2319846.

[2] N. Ekmekçi, H.H. Hacısalihoğlu, and K. İlarslan, Harmonic Curvatures in Lorentzian Space, Bull. Malaysian Math. Sc. Soc. (Second Series), 23 (2000) 173-179

[3] H. Liu, D. Pei, Singularities of a space curve according to the relatively parallel adapted frame and its visualization, Math. Prob. in Engrng., 2013 (2013) 12 pages, http://dx.doi.org/10.1155/2013/512020.

[4] R. Lopez, Differential geometry of curves and surfaces in LorentzMinkowski space, Int. Elec. Journ. Geom., 3 (2) (2010) 67-101.

[5] R. S. Millman, G. D. Parker, Elements of Differential Geometry, Englewood Cliffs, N. J.: Prentice-Hall Inc., 1977.

[6] B. O'Neill, Semi-Riemannian geometry with applications to relativity, Academic Press, New York, 1983.009) 46-49.

[7] M. Özdemir, A.A. Ergin, Paralel frames of non-lightlike curves, Miss. Journ. of Math. Sci., 20 (2) (2008) 127-137.

[8] E. Özyllmaz, Classical Differential Geometry of Curves According to Type2 Bishop Trihedra, Math. Comp. Appl., Vol. 16, No.4, (2011), 858-867.

[9] M. Turgut, S. Yılmaz, Contributions to classical differential geometry of the curves in $E^{3}, S c i$. Magna, 4 (2008), pp. 5-9.

[10] M. Turgut, S. Yilmaz, On the Frenet Frame and a Characterization of Space-like Involute-Evolute Curve Couple in Minkowski Space-time, Int. Math. For., 3, 2008, no. 16, 793 - 801

[11] S. Yllmaz, M. Turgut, A new version of Bishop frame and an application to spherical images, J. Math. Anal. Appl., 371 (2010) 764-776, doi:10.1016/j.jmaa.2010.06.012.

[12] S. Yllmaz, M. Turgut, On the Characterizations of Inclined Curves in Minkowski Space-Time $E_{1}^{4}$, Int. Math. For., 3, 2008, no. 16, 783 - 792

[13] S. Yılmaz, Bishop spherical images of a spacelike curve in Minkowski 3space, Int. Jour. Phys. Scien., 5 (6) (2010) 898-905. 
[14] S. Yllmaz, Contributions to differential geometry of isotropic curves in the complex space, J. Math. Anal. Appl., 374 (2011), 673-680, doi:10.1016/j.jmaa.2010.09.031.

[15] S. Yılmaz, Y. Ünlütürk, A New Version of Bishop Frame and its Application to Smarandache Curves of a Spacelike Curve in Minkowski 3-space, to appear.

[16] J. Walrave, Curves and surfaces in Minkowski space, Ph. D. Thesis, K.U. Leuven, Leuven, Belgium, 1995. 